\title{
A new light of proteomics in cell biology and toxicology
}

\author{
Tiankui Qiao • Xiangdong Wang
}

Received: 31 July 2019 /Accepted: 11 August 2019/Published online: 19 August 2019

(C) Springer Nature B.V. 2019

\begin{abstract}
We expect more studies on proteomicsdominated multi- and trans-omics to paint out a dynamic, multi-level, multi-dimensional picture of molecular mechanisms in the single-cell or targeted cell population to elucidate the regulation of gene-gene, gene-protein, and protein-protein interactions, and define proteomewide strategy of disease-specific biomarker and therapeutic target discovery and development. We hope that the current issue will be the initiation and stimulation of proteome-based investigations to explore molecular mechanisms in cell functioning and responses to drugs.
\end{abstract}

Proteomics is a powerful tool to provide significant value and impact in understanding molecular mechanisms of disease, identify disease-related biomarkers, and validate therapeutic effects (Wang et al. 2006). Proteomics is even more important to improve the clinical outcomes of these patients when proteomic profiles are integrated with clinical phenomes, tissue imaging profiling, and trans-omics. We also believe that proteomics can be a new light in cell biology and toxicology and invited Prof Gyorgy Marko-Varga, a world-leading scientist in translational proteomics, to organize this special issue entitled "Translating Proteomics- and

T. Qiao $\cdot X$. Wang $(\bowtie)$

Zhongshan Hospital Jin Shan Hospital Center for Tumor Therapy, Institute for Clinical Science, Shanghai Institute of Clinical Bioinformatics, Shanghai Engineering Research for AI

Technology for Cardiopulmonary Diseases; Shanghai Medical

College, Fudan University, Shanghai, China

e-mail: xiangdong.wang@ clintransmed.org
Genomics-Medicine into 21st Century Cancer". This will be a starting point for the journal of Cell Biology and Toxicology to focus on developing a deeper understanding of disease-specific proteomic profiles, mechanism-based proteomic regulation, proteindominated cell-cell communication, and interaction between protein with DNA or RNA.

Proteogenomics is an important approach to multidimensionally identify disease-specific functional biomarkers, neoantigens, therapeutic targets, and 3D genome dysfunction. Gil et al. (2019) emphasized the significance of integrating proteogenomics with customized protein sequence databases generated from patient-specific genomic and transcriptomic data to characterize comprehensive molecular functions and target malignant melanoma. With clinical trans-omics (proteogenomics, clinical phenomics, radiomics, metabolomics), mass spectrometry-based imaging can showcase the spatial distribution actions, efficacies, toxic severities, or metabolites of drugs within tissues at single-cell resolution as more efficient and safer strategies for the treatment of melanoma. In addition to the concept that clinical phenomes are integrated with molecular multi-omic profiles (Wang 2018), this comprehensive strategy demonstrates the merge of clinical phenomes in patients with melanoma with tissue imaging and heterogeneity, digital pathology, machine learning, artificial intelligence, biobanking, and sample quality, as well as proteomic profiles. In the present issue, Gil et al. (2019) provides a new insight for better understanding of genes to protein expression and their function in malignant melanoma by integrating disease- 
associated mutations of genes and single amino acid variants of proteins with proteomics-dominated clinical trans-omics, e.g., post-translational modification, pathway signaling, phosphorylation, or acetylomics of targeting proteins. Furthermore, proteogenomic profiles can be characterized for new therapies which aim to discover disease-specific signaling proteins, metabolic enzymes, and tumor antigens and provide strategic guidance of cancer precision therapy (Vasaikar et al. 2019).

Different from multi-omics, trans-omics emphasizes the gathering, spanning, and transparent pointing of various omics layers in different forms, which can hardly have perfect and precise matching. Ansari et al. (2019) presented an example of proteogenomic studies for precision diagnostics and treatment in patients with pancreatic cancer and listed target molecules as "trans" points through which multiple omic layers functionally cross. Those "trans" points can be modeled as predictive nodes connecting multiple dimensional networks of omics layers/datasets and are automatically analyzed by the artificial intelligence system with machine learning capacity (Camacho et al. 2018). With the development of omic measurements, multi-omic profiles in single-cell levels can be characterized and integrated into a single-cell-like system with computerized databases, digitalized informatics of biological elements, and programmed function and signals named "an artificial intelligent single cell" (Zeng et al. 2018). When compensated with cell functional and morphological phenotypes and clinical phenomes, single-cell transomics provide a new approach to systemically understand molecular interactions, communications, and mechanisms in the disease, and can dynamically monitor alterations of single-cell gene/protein expression and networks in patient response to therapies.

A number of protein post-translational modifications play critical roles in the maintenance of functional diversity of the proteome, responsible for the pathogenesis of disease. Colombo et al. (2019) demonstrated that cigarette smoke could increase the carbonylation of a large number of proteins distributed throughout the cell in a concentration-dependent manner. Those carbonylated proteins are involved in protein and lipid metabolism, as well as metabolite and energy production, in the cell cycle and chromosome segregation. In addition, protein neddylation was also found to play regulatory roles in the process of carcinogenesis, intravascular survival, extravasation, and metastasis of cancer through alterations of tissue enzyme activities, epithelial-mesenchymal transit, and cytoskeleton at an early stage (Jiang et al. 2019). Protein modification is a major mechanism by which the cell phenotypes and functions are regulated. For example, tubulin posttranslational modifications can act as important regulators of the microtubule cytoskeleton, properties, and functions, contributing to a number of disorders associated with disease phenotypes, genome profiling, and pathologies (Magiera et al. 2018). By changing cell phenotypes and functions, a large number of protein diversities caused by protein modification can re-program cellular responses to drugs, become new candidates of druggable targets, and result in the development of new therapies. Proteomics-dominated multi- and trans-omics will provide deeper insights for understanding comprehensive mechanisms of modification regulation, evolution, and intervention at the initiation, exacerbation, and chronic processes of the disease.

There are a large number of comprehensive processes from post-transcriptional regulation of gene expression to alterations of proteomic profiles to maintain intracellular and intercellular function. For example, RNAbinding proteomic profiles play regulatory roles in those processes within the cell, responsible for mRNA modification and translation, protein modification, signal pathway, and carcinogenesis. Trendel et al. (2019) developed a new method for the generic purification of protein-crosslinked RNA (XRNAX) to evaluate the composition and dynamics of protein-RNA interactions. XRNAX makes it possible to define the RNA-binding sub-proteomic profiles and protein-RNA interfaces during the interaction between coding and non-coding RNAs and in the processes of mRNA functioning and translation. This also provides more potential and value in studying protein-RNA interaction-associated molecular toxicity within the cell and nucleus when combining XRNAX with other measurements. Cell-cell communication is an old mystery and an example of protein-protein interactions between cells, responsible for keeping biological functions and microenvironmental hemostasis of cells, organs, and intact system highlights a new opportunity (Song et al. 2019). Cell-cell communication can be characterized by ligandreceptor gene expression measured by RNA sequencing in single-cell levels or bulk of target cells. The cell-cell interactions can be also defined through the measurement of small molecule-protein binding and interaction using chemical proteomics on the basis of the fragmentbased covalent ligand discovery. Although the studies 
with proteome-wide covalent ligand discovery were focused on the evaluation of ligand functions according to the affinity degree (Parker et al. 2017), chemical proteomics is also applied for the certain part of cellcell communication by monitoring small moleculereceptor bindings and protein-protein interactions according to the receptor specificity and affinity. Proteogenomic profiles of the single cell by integrating RNA sequencing with chemical proteomics can be a powerful approach for deeper understanding of cellcell communication.

We expect more studies on proteomics-dominated multi- and trans-omics to paint out a dynamic, multilevel, multi-dimensional picture of molecular mechanisms in the single-cell or targeted cell population, to elucidate the regulation of gene-gene, gene-protein, and protein-protein interactions, and define proteome-wide strategy of disease-specific biomarker and therapeutic target discovery and development. We hope that the current issue will be the initiation and stimulation of proteome-based investigations to explore molecular mechanisms in cell functioning and responses to drugs.

\section{References}

Ansari D, Torén W, Zhou Q, Hu D, Andersson R. Proteomic and genomic profiling of pancreatic cancer. Cell Biol Toxicol. 2019. https://doi.org/10.1007/s10565-019-09465-9.

Camacho DM, Collins KM, Powers RK, Costello JC, Collins JJ. Next-generation machine learning for biological networks. Cell. 2018;173(7):1581-92. https://doi.org/10.1016/j. cell.2018.05.015.

Colombo G, Garavaglia ML, Astori E, Giustarini D, Rossi R, Milzani A, et al. Protein carbonylation in human bronchial epithelial cells exposed to cigarette smoke extract. Cell Biol Toxicol. 2019. https://doi.org/10.1007/s10565-019-09460-0.
Gil J, Betancourt LH, Pla I, Sanchez A, Appelqvist R, Miliotis T, et al. Clinical protein science in translational medicine targeting malignant melanoma. Cell Biol Toxicol. 2019. https://doi.org/10.1007/s10565-019-09468-6.

Jiang Y, Liang Y, Li L, Zhou L, Cheng W, Yang X, et al. Targeting neddylation inhibits intravascular survival and extravasation of cancer cells to prevent lungcancer metastasis. Cell Biol Toxicol. 2019;35(3):23345. https://doi.org/10.1007/s10565-019-09472-w.

Magiera MM, Singh P, Gadadhar S, Janke C. Tubulin posttranslational modifications and emerging links to human disease. Cell. 2018;173(6):1323-7. https://doi.org/10.1016/j. cell.2018.05.018.

Parker CG, Galmozzi A, Wang Y, Correia BE, Sasaki K, Joslyn $\mathrm{CM}$, et al. Ligand and target discovery by fragment-based screening in human cells. Cell. 2017;168(3):527-541.e29. https://doi.org/10.1016/j.cell.2016.12.029.

Song D, Yang D, Powell CA, Wang X. Cell-cell communication: old mystery and new opportunity. Cell Biol Toxicol. 2019;35(2):8993. https://doi.org/10.1007/s10565-019-09470-y.

Trendel J, Schwarzl T, Horos R, Prakash A, Bateman A, Hentze MW, et al. The human RNA-binding proteome and its dynamics during translational arrest. Cell. 2019;176(1-2):391403.e19. https://doi.org/10.1016/j.cell.2018.11.004.

Vasaikar S, Huang C, Wang X, Petyuk VA, Savage SR, Wen B, et al. Proteogenomic analysis of human colon cancer reveals new therapeutic opportunities. Cell. 2019;177(4):10351049.e19. https://doi.org/10.1016/j.cell.2019.03.030.

Wang X. Clinical trans-omics: an integration of clinical phenomes with molecular multiomics. Cell Biol Toxicol. 2018;34(3): 163-6. https://doi.org/10.1007/s10565-018-9431-3.

Wang X, Adler KB, Chaudry IS, Ward PA. Better understanding of organ dysfunction requires proteomic involvement. J Proteome Res. 2006;5(5):1060-2.

Zeng Y, Chen X, Gao H, Wang X. An artificial intelligent single cell is part of the cell dream world. Cell Biol Toxicol. 2018;34(4):247-9. https://doi.org/10.1007/s10565018-9433-1.

Publisher's note Springer Nature remains neutral with regard to jurisdictional claims in published maps and institutional affiliations. 